\title{
PROKLA-Redaktion
}

\section{Editorial: Zur (neuen) Wohnungsfrage}

Im November 2013 verkündete die damalige Große Koalition mit viel rhetorischem Tamtam die Einführung der Mietpreisbremse, und der Verband der Haus- und Grundbesitzer befürchtete das Schlimmste: Die Mieten für Neuvermietungen auf maximal 10 Prozent über den ortsüblichen Vergleichsmieten zu begrenzen, sei ein Angriff auf Grundrechte des Eigentums und der Vertragsfreiheit, Neuinvestitionen in den Wohnungsbau würden radikal abgewürgt und bezahlbare Mieten in Ballungsgebieten auf diesem Weg mit Sicherheit nicht erreicht. Zumindest beim letzten Punkt sollte der Verband Recht behalten - wenngleich aus anderen Gründen. Denn während die Investitionen in Wohnbauprojekte keineswegs zurückgingen und der Bau von (hochpreisigen) Eigentumswohnungen neue Höhenflüge erlebte, stiegen die Immobilienpreise und Mieten weiter - unter anderem deshalb, weil Neubauten ohne Regulierung nicht zu leistbaren Mieten führen und die zahnlose Mietpreisbremse nicht nur schwer zugänglich ist, sondern auch zahllose Schlupflöcher aufweist. Dieser erfolglose Versuch einer punktuellen und halbherzigen Reform zeigt beispielhaft zum einen, dass die von Friedrich Engels vor fast 150 Jahren diskutierte "Wohnungsfrage“ wieder ein Thema der Politik geworden ist; zum anderen, dass eine kapitalistisch organisierte Wohnungsversorgung von grundlegenden Interessenskonflikten durchzogen ist, die durch eine partielle Preisregulierung nicht einfach aufzuheben sind.
Engels veröffentlichte seine Überlegungen 1872/1873 in einer Serie von mehreren Artikeln in der Leipziger Zeitung Der Volksstaat. Es ging ihm darum, die Ursachen für die damalige Wohnungsnot breiter Kreise, insbesondere (aber nicht nur) der Arbeiterschaft, zu benennen und aufzuzeigen, dass sämtliche damals diskutierten Reformansätze in Sackgassen führten: „Der Kern sowohl der großbürgerlichen wie der kleinbürgerlichen Lösung der 'Wohnungsfrage' ist das Eigentum des Arbeiters an seiner Wohnung." (Engels 1975, MEW 21: 329). Doch dieses Eigentum hielt Engels für einen Rückschritt, da er es mit der früheren Hausindustrie identifizierte und darin eine Fesselung der Arbeiterschaft gegenüber den Unternehmern sah.

Tatsächlich gehört die staatliche Förderung von Eigentum seit Jahrzehnten zum Grundrepertoire von bürgerlichen (oder auch sozialdemokratischen) Regierungen. Sie galt immer wieder als Befriedungsinstrument, mit dem es vielfach erfolgreich gelang, den „kleinen Leuten“ die Form des Privateigentums schmackhaft zu machen, da das eigene kleine Häuschen doch letztlich nichts anderes darstellen würde als der Besitz von Produktionsmitteln im sehr viel größeren Maßstab. Zuletzt wurde die Eigenheimförderung für junge Familien bei den Sondierungsgesprächen von Union und SPD über eine neue Koalition im Januar dieses Jahres angekündigt. In einzelnen kapitalistischen Ländern war die Bedeutung des Wohnens im eigenen Haus 
allerdings stets sehr verschieden, und in Zeiten überhitzter Preise und fragwürdiger Kredite konnte sich - wie in Spanien und den USA seit dem Jahr 2000 - der Traum vom eigenen Heim binnen Kurzem in einen Albtraum von existenzbedrohender Überschuldung verwandeln.

Auch von Arbeiterkolonien in Form von Werkssiedlungen, wie sie seit Mitte des 19. Jahrhunderts von Großunternehmen wie der Gutehoffnungshütte in Oberhausen mit dem Ziel errichtet wurden, sich eine gefügige Arbeiterschaft zu halten, hielt Engels nichts, und ebenso wenig fanden Selbsthilfeprojekte der Arbeiterschaft wie etwa Baugenossenschaften - Gnade vor seinen Augen. Dass der Staat eingreifen würde, um die Wohnungsnot zu mildern, hielt er ohnehin für illusorisch. Um dieser tatsächlich ein Ende zu machen, sah er somit „nur ein Mittel: die Ausbeutung und Unterdrückung der arbeitenden Klasse durch die herrschende Klasse überhaupt zu beseitigen." (Engels 1973, MEW 18: 213).

Die von Engels diskutierte Wohnungsfrage blieb keineswegs ein auf das 19. Jahrhundert beschränktes Phänomen. Vielmehr stellt sie sich seitdem mit einer gewissen Regelmäßigkeit immer wieder aufs Neue, da Gesellschaften mit einer kapitalistisch organisierten Wohnraumversorgung vor einem grundlegenden Widerspruch stehen. Die Versorgung mit Wohnraum erfolgt in der Regel vermittelt über Marktmechanismen und eingebunden in - zunehmend globale - Prozesse der Kapitalzirkulation. NutzerInnen einer Wohnung müssen daher, sei es als MieterInnen oder als KreditnehmerInnen, über ausreichende Zahlungsfähigkeit verfügen, um die profitable Verwertung des in der Wohnimmobilie investierten Kapitals sicherzustellen. Dieser Umstand steht jedoch immer wieder im Gegensatz zu den Erfordernissen einer ausreichenden
Reproduktion der Klasse der Lohnabhängigen, was insbesondere in Zeiten sinkender oder stagnierender Reallöhne, Renten und Sozialleistungen gilt.

Angesichts dieses gesellschaftlich eingerichteten Widerspruchs haben staatliche Akteure seit Beginn des 20. Jahrhunderts in allen kapitalistischen Ländern jeweils abhängig von den gesellschaftlichen Kräfteverhältnissen sowie der historisch spezifischen Gesellschaftsformation mit diversen Instrumenten in Marktmechanismen interveniert, um die Leistbarkeit des Wohnens sicherzustellen. Anders als von Engels vermutet, wurden im letzten Jahrhundert also durchaus umfassende staatliche Projekte initiiert, wie etwa der kommunal betriebene Wohnungsbau des Roten Wiens der 1920er Jahre, der nicht nur gezeigt hat, dass tiefgreifende staatliche Interventionen möglich sind, sondern gleichzeitig mit dem Ziel der Emanzipation der Arbeiterschaft verbunden sein können. Diese Instrumente waren jedoch immer wieder umstritten und wurden mit dem Vordringen neoliberaler Konzepte ab Mitte der 1980er Jahre vielerorts auch praktischen Angriffen ausgesetzt. Bereits in der alten Bundesrepublik wurde die Deregulierung des Mietrechts, die Abschaffung der Wohnungsgemeinnützigkeit und der Rückzug aus dem sozialen Wohnungsbau beschlossen. Gleichzeitig gingen notleidende Kommunen vermehrt dazu über, ihre Bestände zu privatisieren, da sie hierin einen Rettungsanker zur Konsolidierung ihrer Haushalte sahen. Hierzulande wie anderswo traf eine derartige Politik auf stagnierende Masseneinkommen und eine dramatische Zunahme prekärer Arbeitsverhältnisse, weshalb vor allem seit der globalen Finanzkrise von 2008 an vielen Orten weltweit eine Verschärfung der Wohnungsfrage zu beobachten ist. Steigende Mieten und Wohnungspreise führen 
zu einer Situation, in der es für Haushalte mit geringen und selbst für solche mit mittleren Einkommen immer schwieriger wird, bezahlbaren Wohnraum zu finden.

Doch gehen die neueren Entwicklungen diesmal weit über einen Abbau sozialstaatlicher Sicherungen hinaus. In den letzten Jahren ist eine Tendenz deutlich sichtbar geworden, die sich bereits um die Jahrtausendwende angedeutet hat: Immobilien sind ins Visier der KapitalanlegerInnen geraten. Wachsende Investitionen in den Immobilienmarkt und die Niedrigzinspolitik der EZB, die klassische Kapitalanlageformen unattraktiv werden ließ, verleihen ihnen den Ruf von vorgeblich sicheren Kapitalanlagen und haben zur Entstehung spezifischer Finanzprodukte (z.B. REITs oder REPE-Fonds) geführt, die den Gebrauchswert von Wohnungen weiter in den Hintergrund treten lassen. Im Zuge dieser neuen Möglichkeiten haben deutsche Städte rund um 2000 angefangen, einen großen Teil ihrer Bestände an internationale Investoren zu verschleudern. Diese tragen klingende Namen wie Cerberus - der Höllenhund aus der griechischen Mythologie - und wurden auf diese Art in die Lage versetzt, den früheren sozialen Wohnungsbau nach ihren Verwertungsinteressen zu filetieren und zu segmentieren, also „bessere“ Objekte einzeln zu verkaufen, andere auf Kosten der Mieter zu sanieren und „schlechte“ Objekte sich selbst zu überlassen.

Die Probleme eines zunehmend neoliberalen Regimes der Wohnraumversorgung haben aber auch soziale Proteste hervorgerufen und auf verschiedenen politischen Ebenen zu einem partiellen Umdenken geführt. Sozialpolitische Interventionen in Marktmechanismen erleben in Gestalt von städtebau- und mietrechtlichen Regulierungen oder der Ausweitung der öffentlichen Wohnbauförderung zumindest in
Ansätzen eine Renaissance. Ebenso haben traditionelle Formen der Selbstorganisation wie Wohnungsbaugenossenschaften einen deutlichen Aufschwung erfahren. Umstritten ist jedoch die tatsächliche Durchschlagskraft solcher Neuerungen, da es mehr als fraglich bleibt, ob sie eine dauerhafte Wirkung auf steigende Boden- und Immobilienpreise sowie auf das Niveau der Mieten entfalten können.

Vor diesem Hintergrund werden in diesem Heft Beiträge vorgestellt, die sich mit den polit-ökonomischen Gründen für die Wiederkehr der Wohnungsfrage im Kontext von Finanzialisierung und Neoliberalisierung befassen sowie kritisch Gegenbewegungen und politische Gegenmaßnahmen beleuchten.

Wie allgemein in den damaligen linken Debatten haben Wohnungsfragen in der PROKLA lange Zeit eher ein Schattendasein geführt, galten doch in den ersten Jahrzehnten ihrer Existenz eher der Bereich der Produktion und zyklische Krisen, gewerkschaftliche Kämpfe und sozialistische Parteien als vorrangige Themen. Vereinzelt wurden sie dennoch aufgeworfen, so wenn Helga Fassbinder „Preisbildung, Monopol und Spekulation beim städtischen Boden“" (PROKLA 10/1973) thematisierte, indem sie an die Diskussionen zur Bodenrechtsreform anschloss, die mit den Sanierungen von innerstädtischen Gebieten aufkamen, wenn bisherige Wohngebiete - etwa im Frankfurter Westend - in Geschäftsund Verwaltungsareale umgewandelt wurden, was auch eine erste Welle von Hausbesetzungen auslöste. Anfang der 1980er Jahre traten solche Probleme in der PROKLA abermals stärker in den Vordergrund, als bereits damals eine „neue Wohnungsnot" diagnostiziert wurde, die Renate Hirsch-Borst und Stefan Krätke in PROKLA 45 (1981) veranlasste, sich grundsätzlich mit der „Verwertung des 
Wohnungsbaukapitals" auseinanderzusetzen, während Michael Krummacher im gleichen Heft die Wohnungspolitik der Bundesrepublik unter die Lupe nahm und das Zurückdrängen sozialpolitischer Ziele zugunsten wirtschaftspolitischer Investitions- und Wachstumsziele untersuchte. Schließlich befasste sich Stefan Krätke in PROKLA 61 (1985) angesichts der zunehmenden "Vermarktwirtschaftlichung" mit aktuellen Reformperspektiven wie etwa der Durchsetzung von genossenschaftlichen Modellen. Drei Jahrzehnte später ist es seit Langem überfällig, Probleme des Wohnens und der damit verbundenen Märkte wieder einmal in den Mittelpunkt zu stellen.

Bernd Belina untersucht ausgehend von David Harveys Konzept der „spatiotemporal fixes" und dessen Analyse der zwei großen Krisen - der Blase der New Economy zu Beginn der 2000er und der Subprime Crisis 2007 - die seither eingetretene, im Vergleich zur anglofonen Welt ungleichzeitige Entwicklung des Immobilienbooms in Deutschland. Dabei diskutiert er, fußend auf einer Rekonstruktion von Harveys Ansatz und empirischen Beispielen, auch den Immobilienboom in China und die zusammenhängenden globalen Verschuldungskreisläufe. Knut Unger wirft in seinem Artikel einen tiefer gehenden Blick auf den spezifischen Charakter der Ware Wohnung. Er beschäftigt sich vor allem mit den wirtschaftlichen Transformationen seit den 1990er Jahren und stellt dabei die aktuellen Prozesse der Finanzialisierung heraus: von der Transnationalisierung des Immobilienbesitzes über zentrale Mechanismen der Mieterlössteigerungen hin zu möglichen Gegenstrategien. Eine Analyse der Entwicklung des sozialen Wohnungsbaus in Deutschland wird von Barbara Schönig in ihrem Artikel vorgelegt. Sie diskutiert dabei, inwiefern sich im Zuge nationaler und städtischer
Neoliberalisierungsprozesse sowie der Durchsetzung städtebaulicher und planerischer Leitbilder der Postmoderne ein neues Paradigma sozialen Wohnungsbaus herausgebildet hat. Lisa Vollmer und Justin Kadi widmen sich der Frage, ob sich eine Verschiebung von marktorientierter Aufwertungspolitik hin zu postneoliberaler Wohnungspolitik vollzogen hat. Ihr Artikel diskutiert in vergleichender Perspektive, inwiefern infolge sozialer Proteste in der Berliner und Wiener Wohnungspolitik seit 2008 ein Paradigmenwechsel hin zu einer verstärkten Dekommodifizierung von Wohnraum eingeläutet wurde. Das Spannungsfeld zwischen einem möglichen Bruch mit Neoliberalisierungsprozessen in der Wohnungspolitik auf der einen, und der Gefahr der Einhegung von sozialem Protest auf dem „Terrain des Staates" auf der anderen Seite, wird von Jonathan Diesselhorst erörtert. Sein Beitrag beleuchtet anhand der Berliner Mietenvolksentscheid-Initiative die Implikationen der Präsenz sozialer Bewegungen im Staat im Hinblick auf die Möglichkeiten, emanzipatorische Alternativen zum neoliberalen Urbanismus zu artikulieren. Jenny Künkel analysiert in ihrem Artikel, wie "Armutsmigration“ sowohl als rassistisch-klassischer Diskurs wie als Prozess der Prekarisierung fungiert. Sie zeigt dabei zentrale Akteure und Diskursmuster, Verwaltungspraktiken und Interventionen städtischer Bewegungen auf, um zu zeigen, dass lokale Aushandlungen maßgeblich durch Logiken der inversen Städtekonkurrenz geprägt sind. „Das Haus, wenn vermietet, bringt seinem Erbauer Grundrente" heißt es bei Engels (1973, MEW 18: 270). Mit einem Beitrag zu diesem Thema schließt Ingo Stützle den Schwerpunkt. Die Grundrente markiert ihm zufolge eine Grenze der marxschen Werttheorie. Das private Grundeigentum ist zwar die 
Voraussetzung aller im vorliegenden Heft diskutierten Phänomene; die Grundrente wird jedoch meist nur als Monopolpreis unterstellt, eine Erklärung, die Marx aber gerade ablehnte. Was bedeutet das für eine an Marx orientierte Debatte?

Alle drei Einsprüche des vorliegenden Heftes widmen sich ebenso dem Schwerpunktthema. Inga Jensen und Sebastian Schipper entkräften in ihrem Einspruch gängige Mythen zur Gentrifizierung, die häufig sozio-kulturalistischen Erklärungsmustern folgen, in dem sie weniger prominente Ansätze aus der polit-ökonomischen Stadtforschung vorstellen. Vor dem Hintergrund des Konzepts „Stadtbürgerschaft“ diskutiert Henrik Lebubn die neoliberalen Implikationen sogenannter „Diversity“Programme exemplarisch am „Intercultural Cities Programme“. Philipp Mattern stellt die Frage, inwiefern vor dem Hintergrund von Markt- und Sozialstaatsversagen die Etablierung eines neuen Substandards auf dem Wohnungsmarkt droht.

Aus traurigem Anlass eröffnen zwei Nachrufen das aktuelle Heft: Heiner Ganßmann und Elmar Altvater sind verstorben. Beide waren mit der PROKLA eng verbunden, viele Jahre in der Redaktion aktiv oder, wie Elmar, sogar Gründungsmitglied. In einer der nächsten Ausgaben der PROKLA wird die Redaktion das Werk von Elmar Altvater ausführlicher würdigen, als dies in der kurzen Zeitspanne zwischen seinem Tod und dem Erscheinen dieses Heftes möglich war.

Inga Jensen, Sebastian Schipper, Dorothea Schmidt, Sandra Sieron (für die Redaktion)
Als Gastredaktion haben Inga Jensen und Sebastian Schipper am Schwerpunkt dieses Heftes mitgewirkt. Die PROKLARedaktion dankt ihnen ganz herzlich für ihre Ideen und ihr Engagement.

Anfang Mai 2018 fand die Mitgliederversammlung der Vereinigung zur Kritik der politischen Ökonomie e.V. statt, der die PROKLA herausgibt und die Redaktion und den Redaktionsbeirat wählt. Julia Dück, Stefanie Graefe, Katharina Hajek und Stefan Schmalz standen nicht mehr zur Wahl. Wir bedanken uns für ihre mehrjährige gemeinsame Redaktionsarbeit. Ebenso Alex Demirović und Thomas Sablowski, die zukünftig jedoch den Redaktionsbeirat verstärken - wir freuen uns auf die weitere Zusammenarbeit.

\section{Literatur}

Engels, Friedrich (1973): Zur Wohnungsfrage. Zur Wohnungsfrage, $M E W$ Bd. 18: 209-287.

- (1975):Vorwort zur zweiten durchgesehenen Auflage, $M E W$ Bd. 21: 325-334. Fassbinder, Helga (1973): Preisbildung, Monopol und Spekulation beim städtischen Boden. In: PROKLA 10: 1-33.

Hirsch-Borst, Renate/Krätke, Stefan (1981): Verwertung des Wohnungsbaukapitals und Staatseingriffe im Wohnungssektor. In: PROKLA 45: 47-72.

Krummacher, Michael (1981): Wohnungspolitik in der BRD. In: PROKLA 45: 73-111.

Krätke, Stefan (1985): Gemeinwirtschaftliche Reformperspektiven im Wohnungsbau. In: PROKLA 61: 95-125. 